\title{
Aerial Thermography for Energetic Modelling of Cities
}

\author{
Gabriele Bitelli ${ }^{1, *, \dagger}$, Paolo Conte ${ }^{1, \dagger}$, Tamas Csoknyai ${ }^{2, \dagger}$ Francesca Franci ${ }^{1, \dagger}$ Valentina A. Girelli ${ }^{1, \uparrow}$ \\ and Emanuele Mandanici ${ }^{1, \dagger}$
}

1 Department of Civil, Chemical, Environmental and Materials Engineering (DICAM), University of Bologna, 2 v. Risorgimento, Bologna 40136, Italy; E-Mails: paolo.conte@unibo.it (P.C.); francesca.franci2@unibo.it (F.F.); valentina.girelli@unibo.it (V.A.G.); emanuele.mandanici@unibo.it (E.M.)

2 Department of Building Service and Process Engineering, Budapest University of Technology and Economics, Müegyetem rkp. 3., Budapest H-1111, Hungary; E-Mail: csoknyaitamas@gmail.com

$\dagger$ These authors contributed equally to this work.

* Author to whom correspondence should be addressed; E-Mail: gabriele.bitelli@unibo.it; Tel.: +39-051-209-3104; Fax: +39-051-209-3114.

Academic Editors: Richard Müller and Prasad S. Thenkabail

Received: 19 December 2014 / Accepted: 9 February 2015 / Published: 16 February 2015

\begin{abstract}
The rising attention to energy consumption problems is renewing interest in the applications of thermal remote sensing in urban areas. The research presented here aims to test a methodology to retrieve information about roof surface temperature by means of a high resolution orthomosaic of airborne thermal infrared images, based on a case study acquired over Bologna (Italy). The ultimate aim of such work is obtaining datasets useful to support, in a GIS environment, the decision makers in developing adequate strategies to reduce energy consumption and $\mathrm{CO}_{2}$ emission. In the processing proposed, the computing of radiometric quantities related to the atmosphere was performed by the Modtran 5 radiative transfer code, while an object-oriented supervised classification was applied on a WorldView-2 multispectral image, together with a high-resolution digital surface model (DSM), to distinguish among the major roofing material types and to model the effects of the emissivity. The emissivity values were derived from literature data, except for some roofing materials, which were measured during ad hoc surveys, by means of a thermal camera and a contact probe. These preliminary results demonstrate the high sensitivity of the model to the variability of the surface emissivity and of the atmospheric parameters, especially transmittance and upwelling radiance.
\end{abstract}


Keywords: aerial thermography; radiometric calibration; atmospheric correction; emissivity

\section{Introduction}

The rising attention to energy consumption problems is raising the interest for the applications of thermal remote sensing in urban environments. In the report of the Intergovernmental Panel on Climate Change, it is clearly stated that the most cost-efficient way to decrease $\mathrm{CO}_{2}$ emissions is through the implementation of energy-efficient retrofitting measures in the building sector [1]. Furthermore, in a recent letter to the President of the United States, a consortium of several industrial partners requested that his administration adopt policies to provide householders with reliable and actionable information at a large scale about the energy use of their buildings [2]. It is obvious that efficient building diagnostic tools can make a major contribution to fulfilling these goals.

Thermography, including all of the techniques for the analysis of the infrared radiation emitted or reflected by objects in the thermal infrared region of the electromagnetic spectrum, provides the opportunity to analyze both the thermal characteristics of the materials lying on the ground and many processes related to the exchange of heat between the surfaces; its importance has been demonstrated in a wide range of military, civil, industrial and scientific applications [3,4]. This science can be successfully used also for energy saving purposes in the urban environment (with particular emphasis on housing stocks and industrial areas) involving several types of stakeholders beside householders, such as public agencies and municipalities, urban planners, entrepreneurs in the field of the energy performance of buildings, constructors and real estate agencies.

Thermal remotely-sensed imagery can be widely used in urban landscapes, because it provides a synoptic and time-synchronized grid of temperature data, even of very large areas, and therefore, it permits analysis at different scales of urban building materials, energy use and losses, surface energy budgets and urban heat island effects $[3,5,6]$. In particular, aerial thermographic surveys in urban areas can reach a very high level of spatial detail, covering, at the same time, very large areas if compared to traditional survey techniques $[7,8]$, thus resulting in being very attractive for the evaluation of the energetic performance of buildings at an urban scale. These kinds of applications continue to increase, especially in continental areas or in cold climates, like the UK, Canada and northern United States [2,9]. The results of these studies are usually raster maps of surface temperatures, which can be easily implemented and processed in the Geographic Information System (GIS) for the creation of spatial decision support systems (SDSS) intended for decision makers.

Although, when compared with the past, advances in thermal infrared detectors and techniques have facilitated obtaining high-resolution thermal images over large areas, for a correct quantitative evaluation of land surface temperatures (LST) and the implementation of the derived maps in the GIS environment, several processing phases are required. In addition, an appropriate choice of the instruments to be used and a detailed planning and execution of the surveys are required. In fact, it is necessary to correct the acquired images, taking into account the effects of the acquisition geometry, the topography of the scene, the influence on the measured values of the atmosphere and of the physical-chemical properties of the different materials lying on the ground. Moreover, several additional 
data, besides the high-resolution thermal imagery, are required to perform all of these steps of processing and to compute accurate surface temperatures; among them, multispectral imagery, digital elevation models, atmospheric measurements, ground surveys and GNSS recordings.

From the obtained temperature data of building surface elements, estimations about the energetic quality of the building shell can be assessed. In the EnergyCity project of the Central Europe Program [10], a spatial decision support system has been developed based on GIS maps. In the project, seven cities from Central Europe (Budapest, Bologna, Prague, Munich, Treviso, Velenje and Ludwigsburg) were surveyed by aerial thermography. The processed data were integrated into a GIS tool aimed at the support of decision making in urban development strategies and action programs in the participating cities [11].

Similar actions were carried out in the HEAT (Home Energy Assessment Technologies) pilot project conducted over the City of Calgary, Alberta, Canada. "HEAT represents a Free and Open Source (FOSS) Geomatics solution for residential waste heat monitoring with high-resolution airborne thermal imagery that is intended to: (i) support Canadian urban energy efficiency programs; (ii) evaluate and monitor residential thermal envelopes (i.e., waste heat) using a new Canadian thermal airborne sensor; and (iii) provide free online results and tools to help individuals conserve energy, save their money and reduce their Green House Gas (GHG) footprint." [7].

In the following sections, some theoretical basics of aerial thermography are firstly discussed; then, a detailed description of the processing workflow executed to obtain a georeferenced map of surface temperature in the urban area of Bologna (Italy) is given; finally, the results of the experimentation and some considerations of the applicability of the mentioned techniques are reported.

\section{Basic Theory}

The temperature assessment of surfaces by using thermal imagery involves the modelling of the radiative properties of the surfaces and of their interactions with the surrounding environment [12,13]. In this section, a brief summary of the model is presented. Under the formalism adopted here, small letters indicate the spectral quantities, which are functions of the wavelength, while the broadband quantities, integrated or averaged over the spectral range to which the thermal camera is sensitive, are designated by capital letters.

The investigated surfaces emit thermal radiation $\left(l_{B}\right)$ depending on their temperature, according to Planck's law. As real surfaces are not black-bodies, their radiative behavior is controlled by the emissivity $(\varepsilon)$, which is, in general, a function of the wavelength and of the observing angle. The emissivity is generally lower than one; then, surfaces also reflect part of the radiation received by the sky $\left(l_{d}\right)$ and by the surrounding objects $\left(l_{s r}\right)$. The emitted and reflected radiation coming from the investigated surface, together with the radiation directly emitted by the atmosphere layer $\left(l_{u}\right)$, are the main components of the radiation registered by the thermal sensor $\left(l_{S}\right)$. A simplified equation, written in terms of spectral radiances, can model the described situation,

$$
l_{S}=\tau \varepsilon l_{B}+l_{u}+\tau \rho F l_{d}+\tau \rho(1-F) l_{s r}
$$

where $\tau$ is the atmospheric transmittance along the path between the investigated surface and the thermal sensor, $F$ is the sky view factor representing the portion of the sky that is visible from the surface and $\rho$ 
is the spectral reflectance of the surface, which can be computed as $1-\varepsilon$. As a simplification, the sky view factor is assumed to be equal to unity, which means assuming a negligible contribution from the surrounding objects.

Each thermal sensor registers the radiation within a specific portion of the electromagnetic spectrum $(\Delta \lambda)$; thus, the radiance registered by the sensor is a broadband radiance, which can be defined as,

$$
L_{S}=\frac{\int_{\Delta \lambda} l_{S} s d \lambda}{\int_{\Delta \lambda} s d \lambda}
$$

where $s$ is the relative spectral response function of the sensor. By substituting Equation 1 in Equation 2 and solving for the $l_{B}$ term and after some simplifications, the following equation can be written,

$$
\int_{\Delta \lambda} \tau l_{B} s d \lambda=\frac{\int l_{S} s d \lambda-\int l_{u} d \lambda-\mathrm{P} \int \tau l_{d} s d \lambda}{\mathrm{E}}
$$

The main theoretical simplification leading to Equation 3 regards the broadband surface emissivity E and the broadband reflectance P [14]. In fact, a grey-body behavior is assumed for all of the surfaces, at least within the $\Delta \lambda$ spectral range. This assumption is also suggested by a practical consideration, discussed later, about the estimation of the emissivity values for the different materials, which can be found in the images. Furthermore, the surface is assumed to be Lambertian, which means that the dependence of $\varepsilon$ and $\rho$ on the observing angle is neglected.

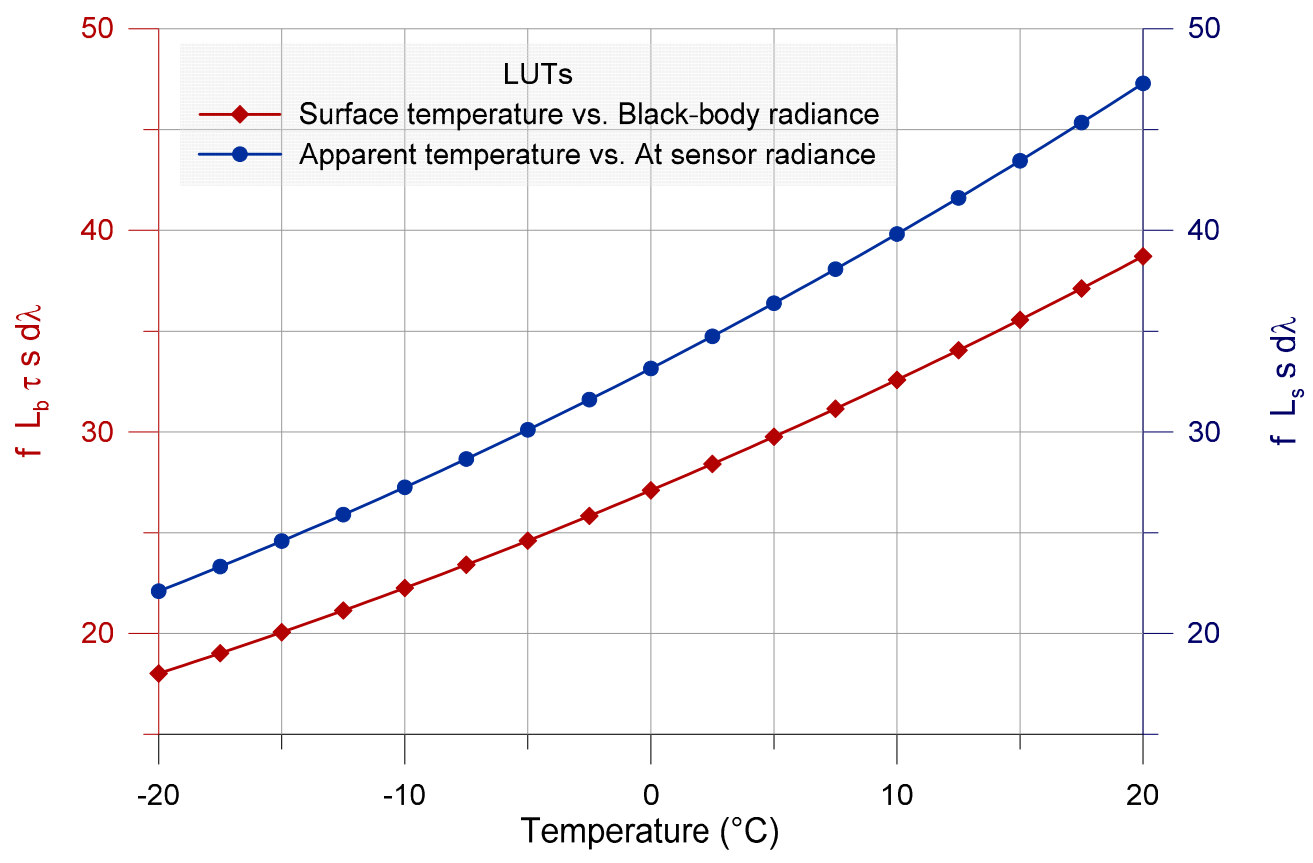

Figure 1. Plot of the look-up tables used to model the relations between the surface temperature and the black-body radiance (red line with diamonds, left vertical axis) and between the apparent temperature and the radiance at sensor (blue line with circles, right vertical axis). Vertical axes are in units of $\mathrm{W} / \mathrm{m}^{2} / \mathrm{sr}$.

To derive the surface temperature from the left side term of Equation 3, a look-up table (LUT) can be built and a second order polynomial relation can be fit (see Figure 1). The temperature range investigated 
by the LUT is to be chosen according to the thermal image statistics and to the expected temperatures. Within this range, the integral of the radiance is calculated numerically at a step of 0.5 degrees, according to Planck's law. The second order polynomial regression results in a residual sum of squares of 0.003 and in a coefficient of determination practically equal to one.

The information obtained from the processed data enables some possibilities for energy analysis of the buildings. The problems related to these applications were analyzed by Allinson [9], proving that "roof surface temperature from the thermal image was complicated by roof material properties, the intervening atmosphere and the surrounding topography. Relating roof surface temperature to insulation thickness was further complicated by loft space ventilation and the outside surface heat balance. The additional data, needed to quantify the results, produced inaccuracies caused by measurement error."

In spite of that, the energetic quality levels of different building elements are often interrelated, because at the time of the construction, the building elements were usually characterized by similar quality. In addition, the expected lifetime of the different building shell elements is similar; thus, often, the same renovation cycles and, thus, the same quality levels can be considered for roofs, facades, windows, etc. This statement can be concluded from the results of the Tabula/Episcope project, where building stock typologies were developed for 20 European countries $[15,16]$. The application of complex renovation measures is often required by support programs requiring similar quality levels for the different elements. Considering all of these aspects, it can be supposed that a high quality roof usually represents a high quality building and vice versa; thus, the roof can be a good indicator for the building as a whole. Based on this hypothesis, indicative information can be obtained from the airborne data. Certainly, this statement can be applied at district levels rather than for individual buildings, where the errors are balanced, providing useful information at this scale for decision makers.

\section{Materials and Methods}

According to the model presented in Equation 3, the radiometric correction procedure to obtain the image of the surface temperature, starting from the image of apparent (at sensor) temperature, has been implemented in the ENVI-IDL environment. The proposed procedure works on raster datasets on a pixel per pixel basis. The required inputs are the image of apparent temperature, the outputs of the radiative transfer simulations in the atmosphere and a raster map of the surface emissivity. The procedure returns a raster map of the corrected surface temperatures.

\subsection{Thermal Images}

An aerial thermal infrared survey over the city of Bologna was performed on 7 March 2011. The survey was carried out by the National Institute of Oceanography and Experimental Geophysics (OGS), in the framework of the Central Europe European Project "EnergyCity" [10]. The thermal infrared frames were acquired using a thermal camera, NEC TS9260, characterized by an un-cooled microbolometer sensor operating in the 6-17 $\mu \mathrm{m}$ spectral range, with a spatial resolution of $640 \times 480$ pixels (with a corresponding field of view of $21.7^{\circ} \times 16.4^{\circ}$ ). The infrared camera was installed with a rigid mounting device on a "Piper Seneca II" aircraft, in combination with an inertial measuring unit (IMU), a video encoder-decoder (VED) and a GNSS receiver. The relative position between the GNSS antenna reference point and the optical center of the thermal camera was accurately measured in 
order to compute with high precision the coordinates of the principal point for each photogram. In order to have optimal coverage, a forward overlapping factor of $60 \%$ and a transversal overlapping factor of $20 \%$ were chosen. In addition, to obtain an average ground sampling distance of $50 \mathrm{~cm}$ and considering a time span between two adjacent shots of $2 \mathrm{~s}$, an average height of the flight of $840 \mathrm{~m}$ and an average speed of the aerial platform of 110 knots were planned. The area of the city of Bologna was surveyed following parallel strips in the W-E direction. The entire thermal survey is formed by 1417 infrared frames.

To carry out the thermal survey, some recommendations about the optimal meteorological conditions, derived from previous studies on the thermal mapping of buildings [9], were followed. The flight was carried out over a few hours after sunset, minimizing the effect of solar irradiation on the surfaces. Furthermore, it was performed in the winter season, during a cold night, with light winds and clear skies, in order to both maximize the differences between the temperatures of the roofs of the buildings and the outside air temperature and to minimize the effects of turbulence at the flight altitude on the stability of the aerial platform.

Simultaneously with the aerial survey, some ground measurements of surface temperature and emissivity were collected, as described in Section 3.4.

The entire processing workflow described in this paper was performed only on a small test area of about $1.06 \mathrm{~km}^{2}$, covered by 30 thermal frames, in order to define the methodology and to evaluate the sensitivity of the model at each step of the processing chain.

The aerial thermal orthophoto-mosaic of Bologna was generated using the Socet Set ${ }^{\mathrm{TM}} 5.6$ digital photogrammetric workstation. The whole process includes several phases: after the project creation, with the indication of the chosen datum and reference system, the images were imported into the project, associating the corresponding camera calibration file (in this case, the only information available about the camera is the focal length and the principal point coordinates). After these steps, the image exterior orientation can be solved by means of a photogrammetric triangulation procedure, using tie and control points. The municipality technical cartography of Bologna at 1:2000 scale was used as the cartographic base for the individuation of the control points, which usually are the edges of buildings' roofs; a large number of points was used, achieving an RMS of 1.5 pixels and residuals of some centimeters for the control points.

After the image orientation, the orthophoto-mosaic, characterized by a ground sampling distance of $0.5 \mathrm{~m}$, was generated (Figure 2) by using a digital surface model (DSM), derived from an aerial LiDAR survey carried out in 2009 and provided by the Municipality of Bologna, with a post spacing of $1 \mathrm{~m}$.

\subsection{Atmospheric Correction}

In the model presented in Equation 3, three quantities are related to the atmosphere and its physical condition at the time of the flight: the transmittance $\tau$ along the path from the surface to the on-board sensor, the upwelling radiance $l_{u}$ emitted by the atmospheric layer in the observing direction and the downwelling radiance $l_{d}$ emitted by the whole atmosphere toward the surface and reflected in the observing direction. All of these spectral quantities were computed by the Modtran 5 radiative transfer model [17]. 


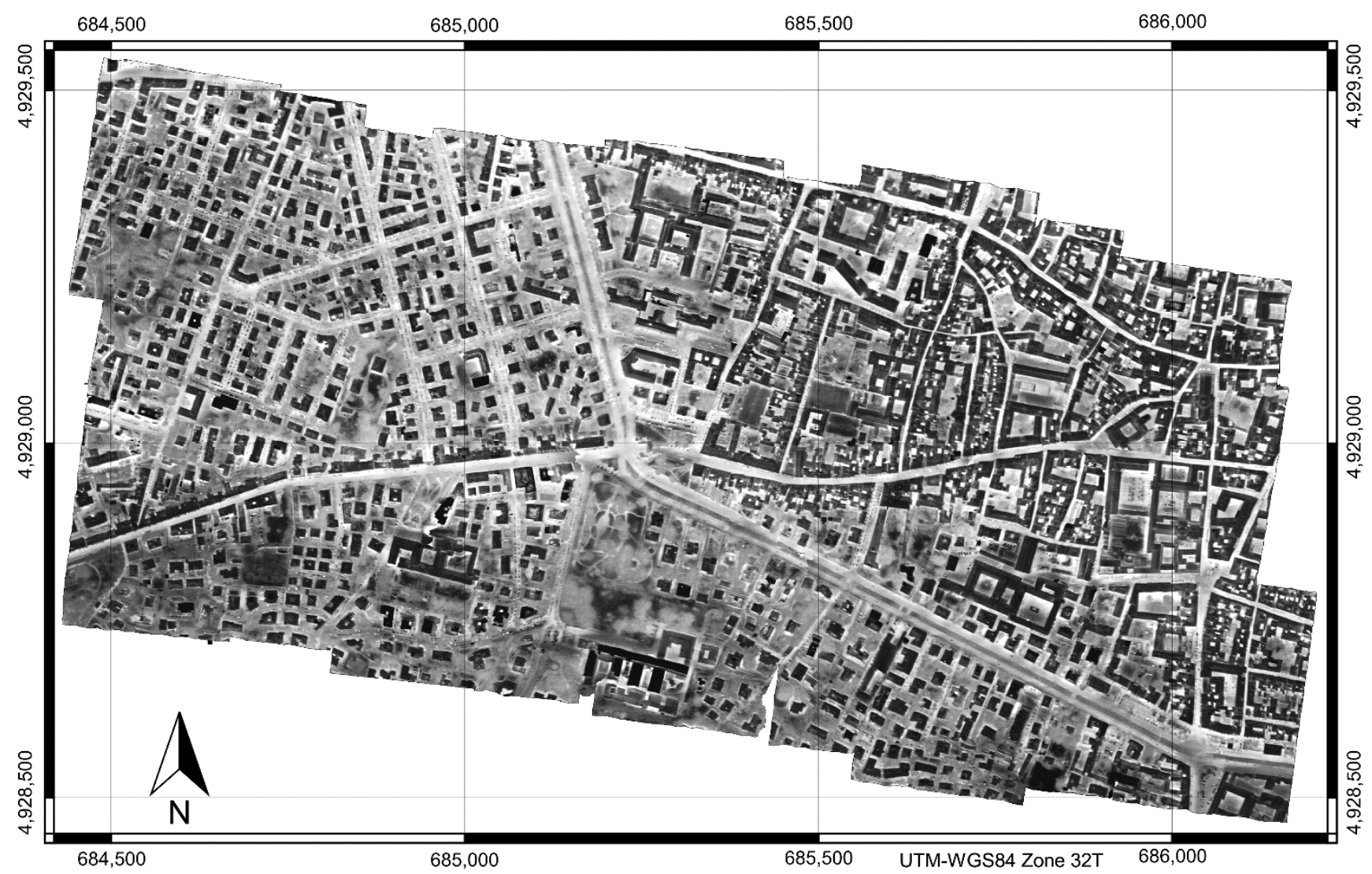

Figure 2. Orthophoto-mosaic of 30 thermal images acquired over the test area in Bologna.

A rigorous parameterization of the model requires measured vertical profiles of the air temperature, pressure and humidity and the volume mixing ratio of each molecular species composing the atmosphere. Of course, such detailed knowledge is normally unavailable. For the present study, however, three different parameterization of the Modtran model were tested and compared. The first run used the profiles obtained by a daily radiosonde launched at 00:00 UTC from the station of the Regional Agency for Environmental Protection (ARPA) at San Pietro Capofiume, located about $30 \mathrm{~km} \mathrm{NE}$ of Bologna. It provides vertical profiles of temperature, pressure, relative humidity and wind speed from the ground to an altitude of about $20 \mathrm{~km}$. The second run used instead the profiles obtained by a MODIS Terra second level product [18], based on an image acquired at 20:30 UTC, about one hour later than the thermal flight. MODIS data contain also information about the total precipitable water vapor and the total ozone burden in the air column. Finally, the third run of the model simply used the mid-latitude winter (MLW) pre-defined profile, which would be the most appropriate choice, if no other data were available. A comparison between the vertical profiles of air temperature used in the three Modtran runs is shown in Figure 3.

For the calculation of the upwelling radiance and the transmittance, the radiative transfer was simulated between the ground and the height of flight. Instead, for the calculation of the downwelling irradiance, the simulation was performed from a height of $100 \mathrm{~km}$ to the ground level, and it has been repeated, varying the impinging angle from 0 to 90 degrees by a step of 5 . For these simulations, when using the sounding data, the vertical profiles were completed with the MLW model above a height of $20 \mathrm{~km}$; instead, the total precipitable water vapor value was used when using MODIS Terra data. 


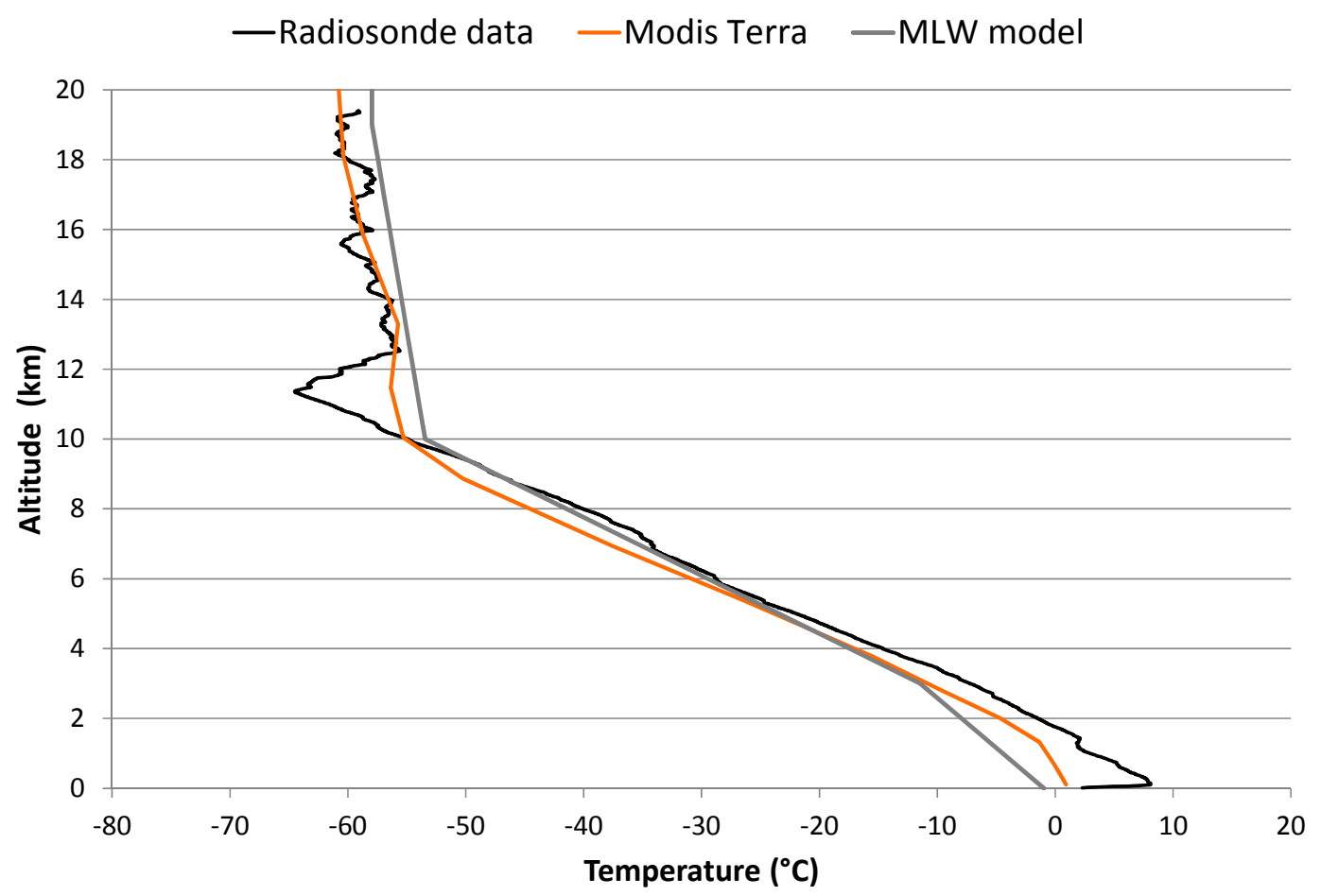

Figure 3. Comparison between the vertical profiles of air temperature derived respectively by radiosonde data (black), by the MODIS Terra second level product (orange) at the same location of the meteorological station and by the mid-latitude winter pre-defined profile (grey).

The differences in the resulting broadband parameters, following the three approaches in the parameterization of the Modtran model, are summarized in Table 1, where broadband radiances are formally defined as in Equation 2, and the broadband transmittance $T$ is computed as the integral average of the spectral transmittance, weighted by the instrument filter function $(s)$ and by the black-body radiance $\left(l_{B}\right)$. When analyzing those differences, the spatial and time distances between the sounding data and the MODIS ones must be considered.

Table 1. Broadband atmospheric parameters computed by Modtran 5 simulations, using the atmospheric profiles of the radiosonde data, of the MODIS Terra image and of the mid-latitude winter (MLW) predefined model.

\begin{tabular}{cccc}
\hline & Radiosonde & MODIS Terra & MLW Model \\
\hline Central wavelength $(\mu \mathrm{n})$ & 10.58 & 10.58 & 10.58 \\
Spectral range $(\mu \mathrm{n})$ & $6-17$ & $6-17$ & $6-17$ \\
Transmittance $T$ & 0.7957 & 0.8052 & 0.8046 \\
Upwelling radiance $L_{u}\left(\mathrm{~W} / \mathrm{m}^{2} / \mathrm{sr} / \mu \mathrm{m}\right)$ & 1.2868 & 1.0969 & 1.0401 \\
Downwelling radiance $L_{d}\left(\mathrm{~W} / \mathrm{m}^{2} / \mathrm{sr} / \mu \mathrm{m}\right)$ & 2.0037 & 1.8243 & 2.0068 \\
\hline
\end{tabular}

\subsection{Emissivity Map}

To produce an emissivity map suitable for the radiometric correction of the thermal image, the major roofing materials in the investigated area were identified, and for each material, a different emissivity value was assigned [19]. 
To distinguish among the major roofing material types, an object-oriented supervised classification was applied on a WorldView-2 multispectral image acquired in 2011 (see Figure 4), which provides 8 spectral bands at a resolution of $2 \mathrm{~m}$, together with the raster DSM, resampled at a resolution of $0.5 \mathrm{~m}$, and a DTM (digital terrain model), at a resolution of $5 \mathrm{~m}$. The object-oriented image analysis was performed with the software eCognition 8 . Before the classification procedure, the satellite image had been radiometrically calibrated and orthorectified. The object-oriented classification consists of two steps: firstly the segmentation, which divides the image into continuous and homogeneous segments, called image objects; and secondly, the classification of the objects generated, using not only the radiometry, but also information, such as texture, shape and context. In this work, at first, the objects corresponding to the buildings were identified; then, they were distinguished depending on their roofing material.
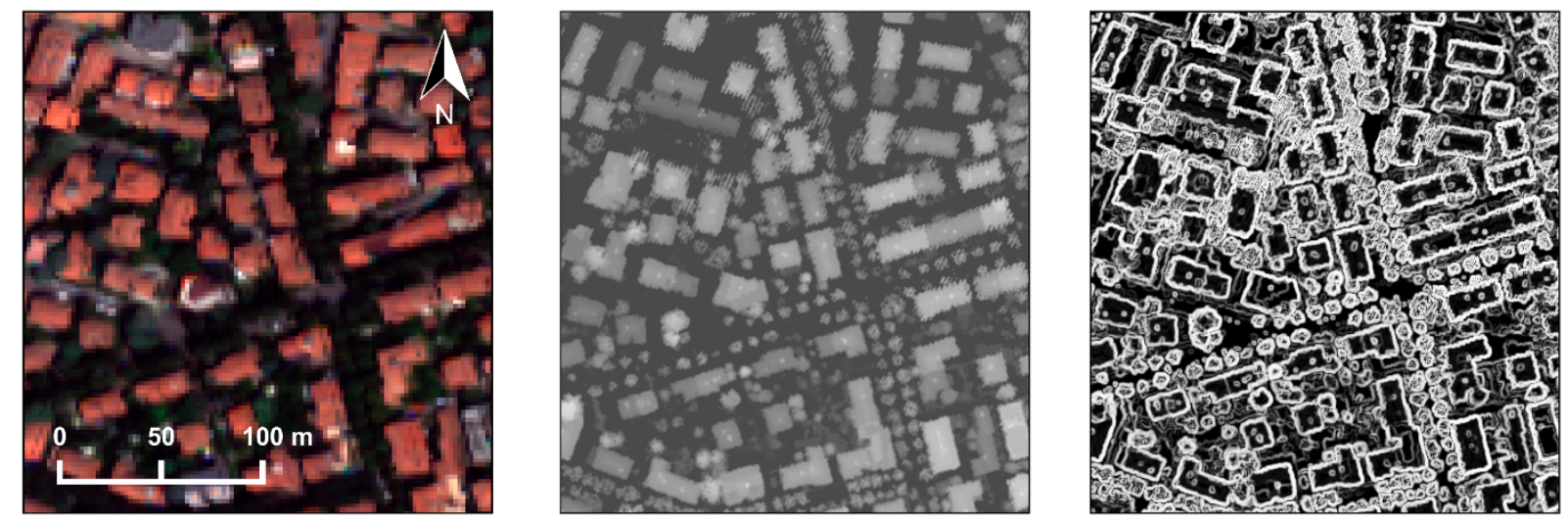

Figure 4. On the left, an RGB color composite of the WorldView-2 image; in the middle, a grey-scale visualization of the DSM; on the right, a visualization of the slope layer derived by the DSM.

A specific feature of buildings is the sudden change in elevation at their borders; in addition, buildings usually have a different elevation than the surrounding environment; therefore, a surface calculation algorithm, which works out slopes, was applied to the DSM. The slope layer has high contrast, and the steep and flat areas are easily recognizable. Thus, the segmentation was applied on this layer using the "contrast split segmentation" algorithm. In particular, two segmentation steps were necessary: the first to get the steep slope objects; the second to separate those with a medium slope from those with very steep ones. This segmentation based on slope information allowed the extraction of objects whose shape is very close to the real footprint of the buildings (see Figure 5). This would have been difficult if a more common multi-resolution segmentation, based on multispectral data only, had been applied. In fact, because of unavoidable artefacts introduced by the orthorectification, the extension of some roofs is greater than the real one.

After obtaining the image objects, a masking classification approach was followed, in order to identify the objects that certainly do not belong to the general class "building". Firstly, vegetated areas have been excluded by means of the NDVI index. Secondly, due to the availability of a DTM of the study area, for each image object, the actual height was calculated by a subtraction operation between DSM and DTM. Then, a threshold was applied to distinguish buildings from other cover types. Finally, to refine this building extraction, the algorithm "find enclosed by class" was applied, so that the 
unclassified objects totally surrounded by objects already classified as buildings were assigned. Furthermore, a geometric rule was used to clean out very small objects.
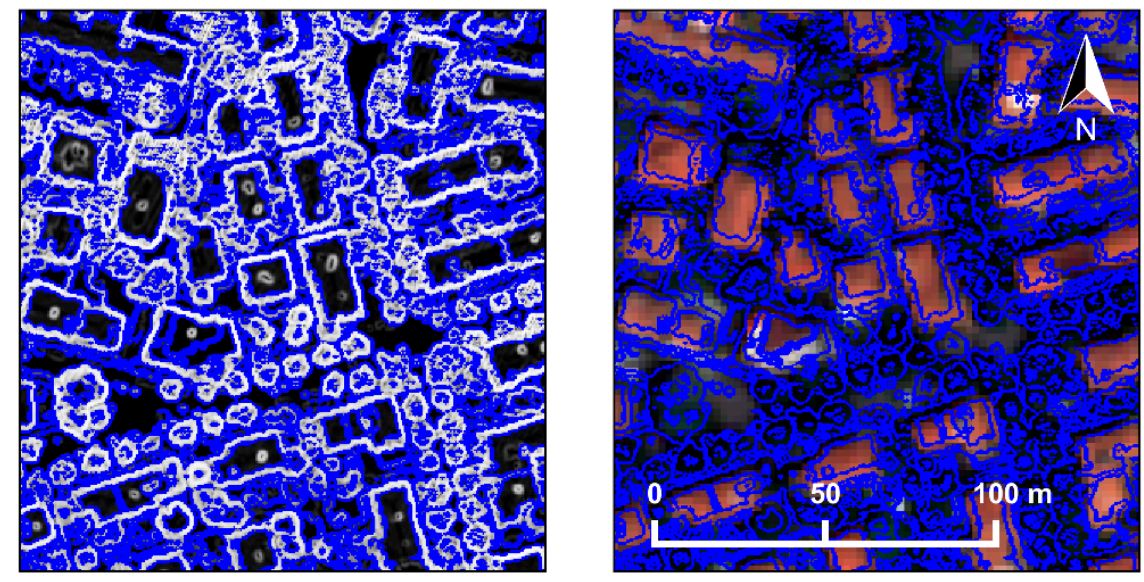

Figure 5. The result of the segmentation procedure plotted over the slope layer (left) and over the RGB composite (right).

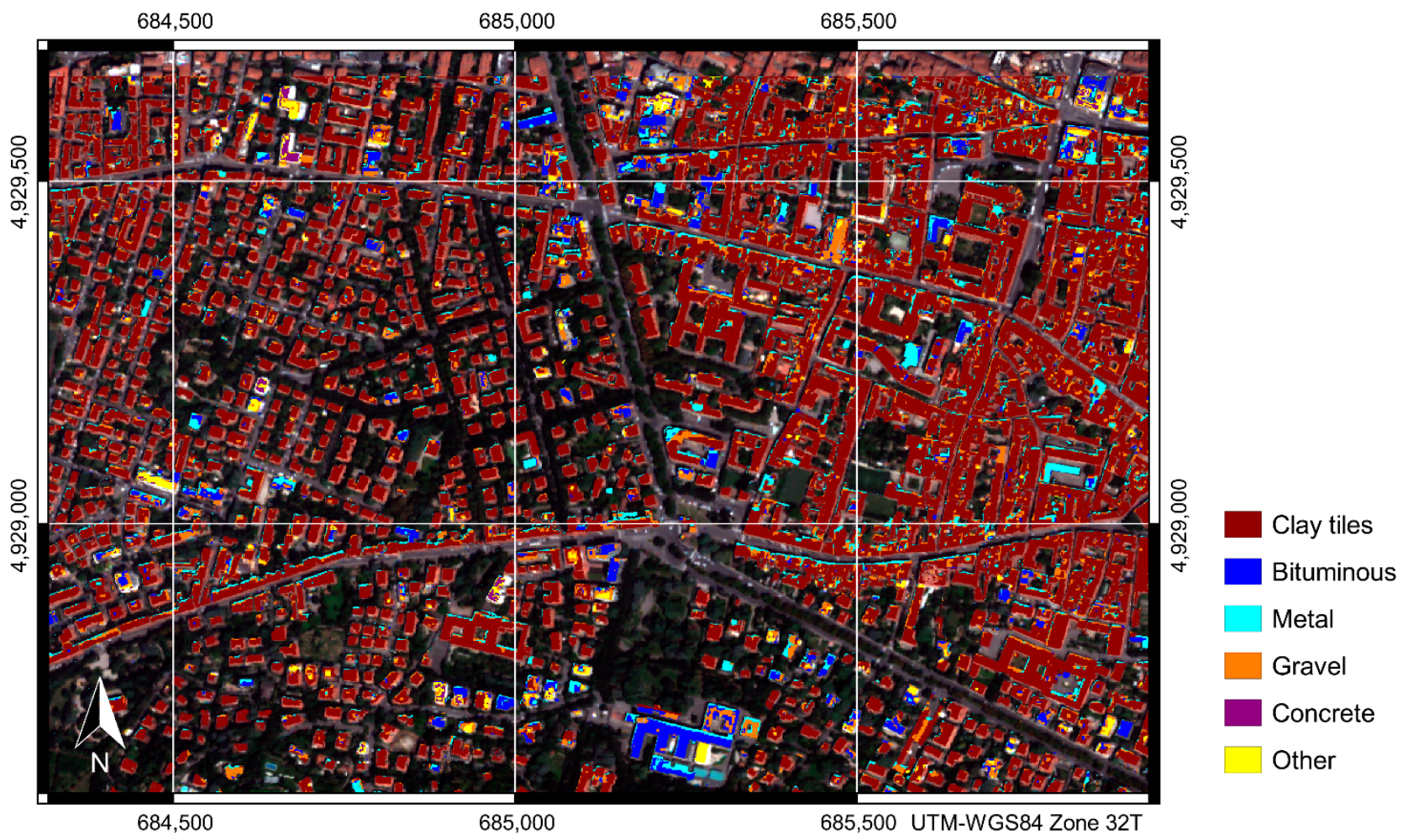

Figure 6. The final classification of the roofing materials of a portion of Bologna, obtained by a supervised object-oriented classification, applied on a WorldView-2 multispectral image together with digital elevation models.

To exploit spectral information for the final distinction of the buildings according to their roofing material, a multi-resolution segmentation, based on the eight bands of the WorldView-2 image, was performed on the previously extracted "building" class. As shown in Figure 6, these new objects were assigned to six informative classes, bituminous, concrete, clay tiles, gravel, metal and other. The 
classification algorithm evaluates the membership of all of the image objects to each class in a selected list. In this case, the membership functions were automatically generated through the selection of training samples for each class. These samples were derived from existing digital cartography and from special surveys.

The last step is the assignment of the emissivity values to each class. In fact, this is a very critical point, because a small variation in emissivity results in a relevant variation in the corrected temperature. In the present study, a direct measure of the emissivity for bituminous and gravel roofing surfaces was performed during a special survey, by means of a thermal camera and a contact probe thermometer. For the other roofing materials, the emissivity values were set according to data published in the literature [19-22]. The values used for the present study are summarized in Table 2.

Table 2. Emissivity values for the roofing types detected in the classification. These values were derived partly from literature data and partly from special surveys (see the discussion in the text).

\begin{tabular}{cc}
\hline Roofing Material & Emissivity Value \\
\hline Bituminous & 0.91 \\
Clay tiles & 0.88 \\
Concrete & 0.90 \\
Gravel & 0.96 \\
Metal & 0.70 \\
Other & 1.00 \\
\hline
\end{tabular}

\subsection{Ground Surveys}

Simultaneous to the aerial survey, some ground measurements of surface temperature and emissivity were collected, to be used as ground truth data for the assessment of accuracy in radiometric correction (Table 3). The locations chosen are mainly squares or open areas, easily recognizable on the thermal mosaic, with homogeneous pavement materials. The geographic positions of targets were computed by post processing techniques using geodetic GNSS receiver recordings, in combination with data from a permanent GNSS stations, while the surface temperature and the emissivity values were derived using a thermal camera FLIR P620, characterized by an infrared sensor with a spatial resolution of $640 \times 480$ pixels operating approximately in the same spectral range of the infrared sensor used for the aerial infrared survey.

Table 3. Apparent temperature derived from the uncalibrated aerial thermal images and emissivity and temperature measured on the ground at the five locations selected for the field surveys. $\mathrm{T}$, transmittance.

\begin{tabular}{ccccc}
\hline Site & Material & Apparent T & Emissivity & Measured T \\
\hline BL01 & cobblestones & -0.2 & 0.91 & 5.3 \\
BL02 & concrete & 0.8 & 0.91 & 5.4 \\
BL08 & asphalt & 0.7 & 0.94 & 8.1 \\
BL10 & asphalt & -0.5 & 0.93 & 3.7 \\
BL11 & stoneware tiles & 0.8 & 0.81 & 8.3 \\
\hline
\end{tabular}




\subsection{Energy Model}

Aerial survey is the quickest and most efficient way of collecting building-related data. However, the usability of such information is limited, as explained above. Primarily, aerial thermographic images give information about the distribution of the roof temperatures and, secondarily, about the heat losses through the facades, as can be noticed in the narrow band around the building outline, but this is only indicative. Generally, the major areas of the applications of the aerial thermographic roof images are:

- Determination of the roof temperature of buildings, which provides accurate results for buildings with heated top-floors only;

- Discovering hot spots; some weak points of a single building, particularly on the roof;

- An indicative classification of buildings based on the relative roof temperature.

In the spatial decision support system of the EnergyCity project, an indicative energy model was applied. It is not the aim of this paper to present the energy model in detail, so only a short overview will be given. The model was based on the so-called relative roof temperature. The roofs were classified into five classes based on their roof temperature compared to the city average value. The upper and lower limits of the ranges were different for the seven cities, because of the different climates and construction types.

From the aerial survey, it is possible to determine the building outline, the perimeter and the covered ground area. The average building height can be obtained from other data sources, such as the comparison between the digital surface model and the digital elevation model. These three types of numerical information are essential to calculate the most important geometric data for energy calculations: the heated volume $(\mathrm{V})$ and the total exposed surface ( $\left.\Sigma \mathrm{A}_{\text {surface }}\right)$. The specific heating energy consumption is calculated for the five temperature ranges, practically defining five heating energy consumption ranges. The consumption ranges were determined on the basis of typical energy consumption values obtained from other building stock databases from the city.

Not only the final heating energy consumption, but also the primary energy consumption values can be estimated by the SDSS, taking into account the primary energy factors laid down in the national building regulations. Similarly, the $\mathrm{CO}_{2}$ emission is determined from the final energy value and the $\mathrm{CO}_{2}$ emission factor of the used energy source. The used energy source was extracted again from other data sources.

As a conclusion, the energy model is partly based on the roof temperatures, some geometric data extracted from the digital cartography and the aerial measurements, but it is inevitable to apply other types of additional data.

\section{Result and Discussion}

All of the data described in the previous section were processed by the ENVI-IDL procedure, and the results of the different parameterizations were compared, to quantify the impacts of each element of the processing chain. As can be seen in Figure 7, the statistics on the corrected temperatures derived by computing the atmospheric parameters using the different vertical profiles presented in Section 3.2 were compared firstly. In order to compare the temperature maps derived from the different atmospheric 
profiles, pixels with a surface temperature below $-10{ }^{\circ} \mathrm{C}$ or above $+10^{\circ} \mathrm{C}$, corresponding in each image to about $5 \%$ of the total number of pixels, were masked out.

The mean roof temperature obtained using the sounding profiles is $-0.7{ }^{\circ} \mathrm{C}$, while the mean value using the MODIS data is $+1.0{ }^{\circ} \mathrm{C}$, and it rises to $+1.6{ }^{\circ} \mathrm{C}$ when the standard MLW model is applied. The standard deviation is more stable and ranges from $2.5{ }^{\circ} \mathrm{C}$ with the MLW model to $2.7{ }^{\circ} \mathrm{C}$ with the sounding profiles. The main difference in the results derived from the sounding profiles with respect to the one obtained using MODIS data is related to a thin layer of thermal inversion in the measured vertical profile of the temperature, located between the ground and a height of $200 \mathrm{~m}$, which is not detectable in the MODIS one (see Figure 3).

It must be noted here that lateral variations and micro-scale patterns of the air temperature in the urban environment were neglected in all of the computations performed. These patterns include temperature differences between urban canyons and open spaces (as large squares or parks) [23] and also horizontal and vertical variations inside urban canyons [24]. Such a simplification is often necessary because of the huge amount of ground measurements that would be required to model all of these complex effects.

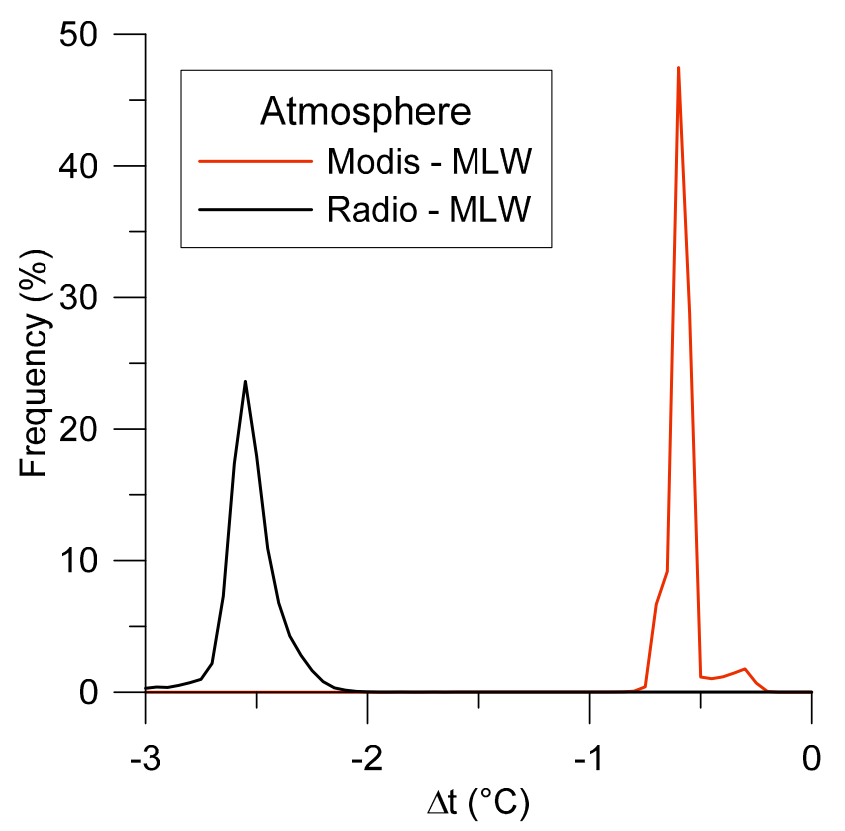

Figure 7. Temperature differences among the corrected images obtained using the atmospheric parameters derived from sounding and MODIS data with respect to the standard MLW profile.

To evaluate the sensitivity to emissivity variation, a test was performed by decreasing the emissivity values reported in Table 2 by about 1\%. Clearly, the effect is an overall increase of the retrieved temperatures, by about $0.3{ }^{\circ} \mathrm{C}$ on average. The effects on each class are shown in Figure 8 . The larger variance of the metal class $(0.017)$ corresponds to a larger variance of its surface temperature, and it may be explained by a greater variability of the roofing material types belonging to this class. In a pioneer study about aerial thermography in urban areas, Artis and Carnahn [25] concluded that the emissivity variations over the most common roofing materials have a minimal effect on the retrieved temperatures. However, they came to this conclusion because of the substantial homogeneity of roofing materials in 
the investigated area. Nowadays, on the other hand, the variety of roofing materials in modern towns is expanding, as the number of design solutions has been increasing since the 1970s. The results presented here indeed demonstrate that the impact of emissivity variation cannot be ignored any longer.

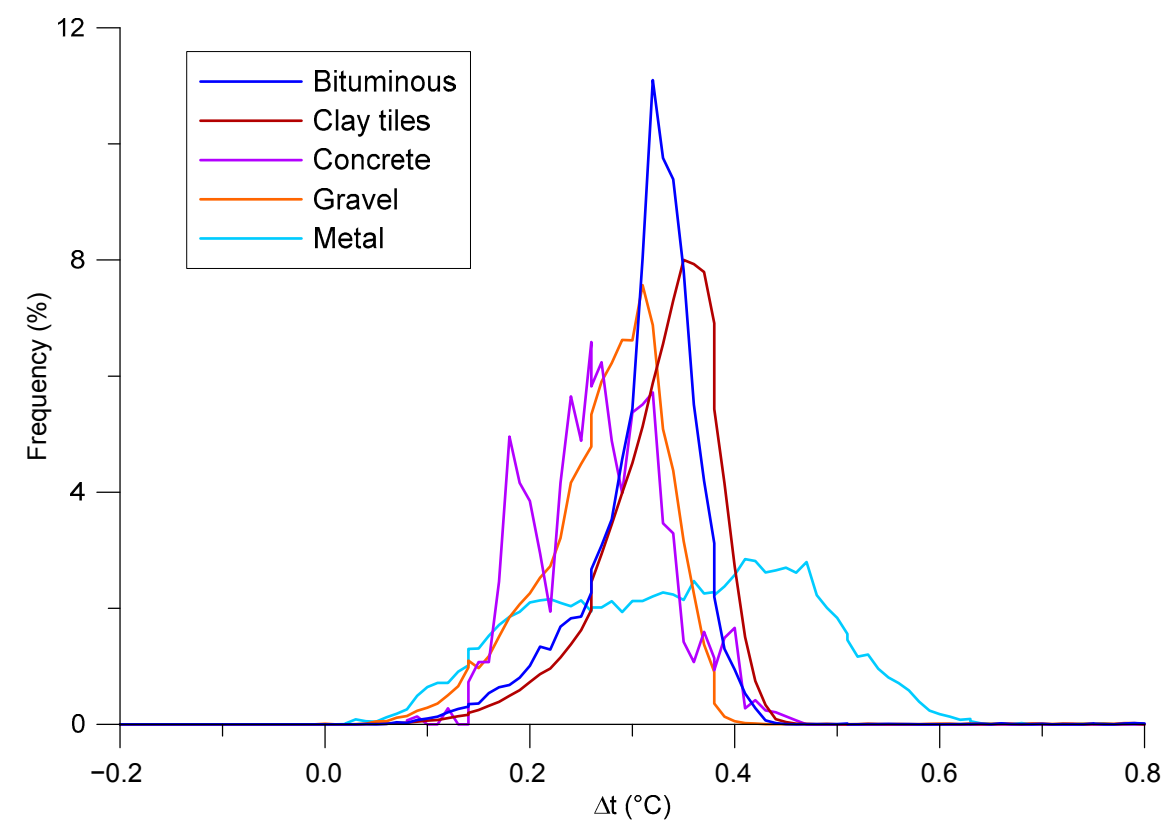

Figure 8. Statistics on the temperature differences for each class, resulting from a decrease by $1 \%$ of the emissivity values listed in Table 2 .

Retrieval of LST from IR data requires, indeed, an accurate knowledge of the emissivity of the surfaces [13], which is controlled by several factors, such as chemical composition, structure and roughness. Furthermore, it can be altered by the possible presence of dew.

As described in Section 2, the thermal infrared radiation acquired by a sensor is a function of, among others, the temperature and emissivity of the surface. Their simultaneous estimation is an ill-posed inversion problem, because the unknowns always exceed the equations. Even with a multispectral infrared sensor with $\mathrm{N}$ thermal bands, in fact, any separation method has to solve $\mathrm{N}+1$ parameters ( $\mathrm{N}$ emissivity values +1 LST) with $\mathrm{N}$ equations. Thus, an a priori assumption or an empirical relation must be introduced to solve the system [26]. On this basis, several temperature/emissivity separation methods were developed to be used with both multispectral and hyperspectral infrared sensors [21,27]: among the most popular, the reference channel method, the emissivity normalization, the alpha residual, the temperature/emissivity separation (TES) and the day/night method. The last one, adopted for MODIS products, uses two images to increase the number of equations, avoiding the ill-posed problem.

All of these separation methods usually assume that the contribution of the atmosphere to the recorded radiance has been already removed [13,26,27]. Equation 3, however, shows that separating atmospheric and emissivity corrections in two steps would require further simplifications (e.g., neglecting the downwelling radiance).

In the present study, the aerial camera acquires only a single infrared band; thus, an alternative approach was adopted: the emissivity values of surfaces were assigned on the basis of a classification of roofing materials from a high resolution satellite image (as described in Section 3.3). The emissivity values were estimated, for each class, by means of direct measurements or other data sources. The main 
disadvantage of this approach is related to the number of direct measurements on roofs, which are often limited because of logistic problems.

As a first attempt of validation, the temperature values obtained from the corrected images were compared with the surface temperatures measured in the field. Temperature differences between the measured and computed values at the five locations are shown in Table 4 . In the first column, the differences obtained by considering temperatures from uncorrected images are shown and prove the relevance of the calibration process. As can be seen, all of the parameterizations of the radiative transfer model produced an average underestimation of the surface temperature. Surprisingly, the worst results were obtained using the radio sounding profile, but this fact can be explained considering that the profile was measured $30 \mathrm{~km}$ away from Bologna in the countryside, so it is probably not representative of the spatial variations of atmospheric transmissivity over urban areas [5]. Furthermore, Jacob et al. [28], performing a sensitivity study on thermal data acquired over a rural area in France, inferred a sensible bias in the retrieved temperature when the profile was measured about $30 \mathrm{~km}$ from the scene, even if they were not able to quantify its amount precisely. They explain this fact in terms of the effects of the topography and spatial variability of the atmosphere.

Table 4. Temperature differences between the measured and computed values at the five locations where ground surveys were performed. The comparison was made with temperatures computed using different sets of parameters for the radiative transfer model (mid-latitude winter, MODIS data, radiosonde profile; see Section 3.2 for details). Furthermore, the differences between measured and apparent temperatures are reported in the first column for reference.

\begin{tabular}{clccc}
\hline Site & \multicolumn{4}{c}{ Atmosphere Model Parameterizations } \\
\hline & None & MLW & MODIS & Radio \\
BL01 & -5.5 & -1.0 & -1.6 & -3.4 \\
BL02 & -4.6 & 0.2 & -0.3 & -2.1 \\
BL08 & -7.4 & -3.9 & -4.5 & -6.2 \\
BL10 & -4.2 & -0.7 & -1.3 & -3.1 \\
BL11 & -7.5 & 2.3 & 1.9 & -0.1 \\
mean & -5.8 & -0.6 & -1.2 & -3.0 \\
\hline
\end{tabular}

The use of MODIS for the parameterization of the atmospheric correction models is discussed in several works dealing with images acquired in the reflective part of the electromagnetic spectrum. Conversely, the use of MODIS atmosphere data for thermal images is still poorly investigated. For instance, Jiménez-Muñoz et al. [18] published the results of some tests in rural areas in Spain using medium-resolution satellite images. They found that average differences between surface temperatures derived using MODIS data and local radio sounding profiles do not exceed $1{ }^{\circ} \mathrm{C}$. However, they do not compare these temperature values with field measurements. The results obtained in the experimentations presented here show a slightly worse performance with respect to the use of the general MLW model. This fact may be due to the loose description of the strata close to the surface, which provide the highest contribution, especially in the case of airborne sensors. Clearly, further investigations and a wider collection of test sites are required to reach more reliable conclusions. 
The temperature dynamic ranges observed (Table 4) are wider than those reported by the mentioned studies, very likely because of the greater complexity of urban environments. The largest discrepancies were observed for the BL08 and BL11 locations. It is rather difficult to distinguish the contribution of each source to the final error. Emissivity plays a primary role in the error resulting at a specific location, both because emissivity itself is a source of error and because different values can mitigate or magnify errors on atmospheric parameters.

The final roof temperature data can be exported to a building database of heat losses or a building energy model. The spatial decision support system of the EnergyCity project combines mapping functionality with energy efficiency measures to delineate and quantify actions and strategies to reduce $\mathrm{CO}_{2}$ emissions and energy usage in the project cities. The system uses a mapping tool to display heat loss maps representing the baseline $\mathrm{CO}_{2}$ emissions in the cities of the project, as well as all other mapping outputs (Figure 9).

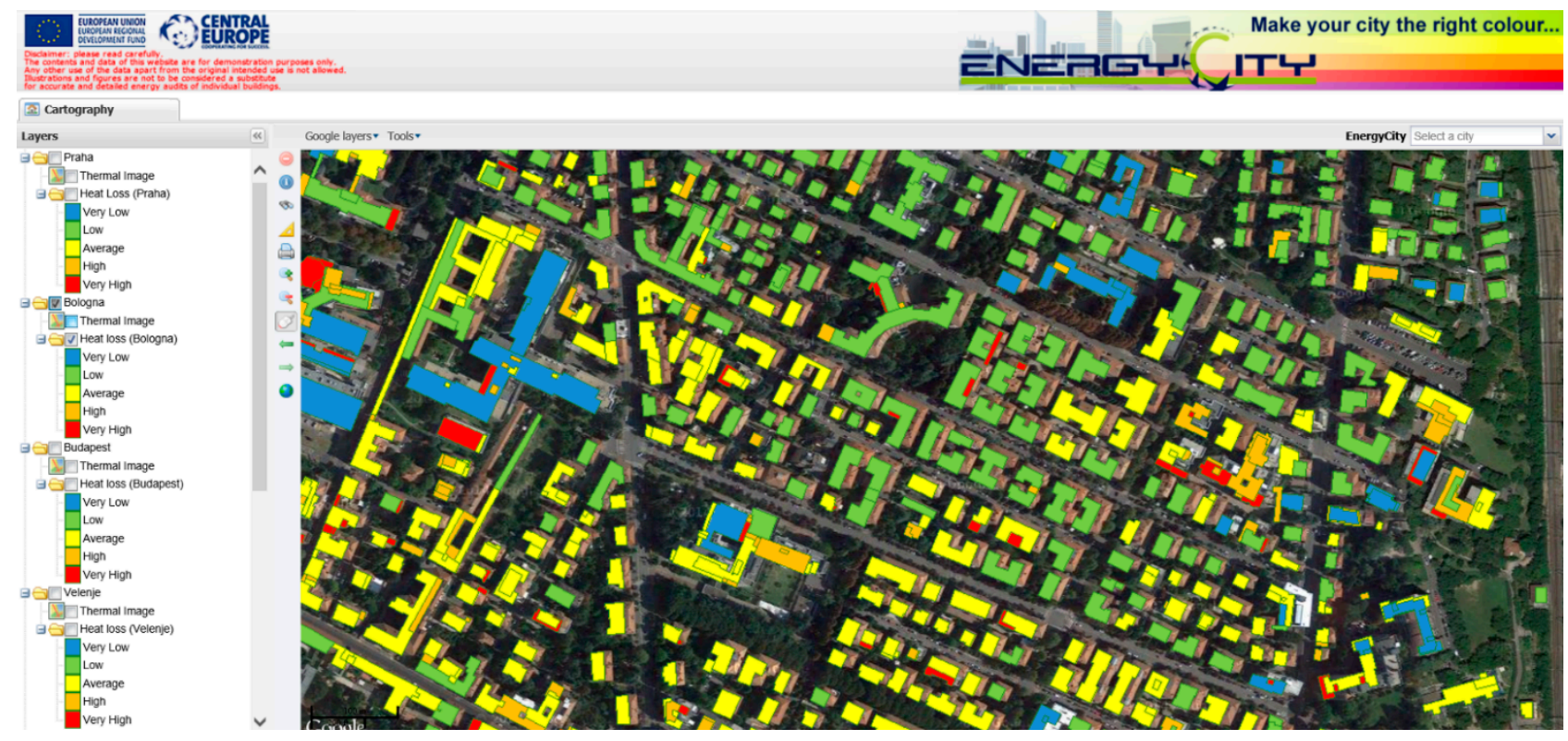

Figure 9. Energy classification of buildings in the spatial decision support system (SDSS) tool developed within the EnergyCity Project.

\section{Conclusions}

The present study investigates the use of aerial thermal imagery for the evaluation of the energy performance of buildings, and it is based on a case study in Bologna (Italy). In particular, the main steps required to measure the surface temperature of roofs were examined, evaluating quantitatively the influence of atmosphere and surface emissivity on the retrieved values.

Even if the physical model behind the radiometric calibration of thermal imagery is well established, its practical implementation requires several ancillary data, whose quality has a strong influence on the quality of the final products. The approach discussed here requires a model of the atmosphere at the time of the thermal flight, a digital surface model and a description of the surface materials in order to derive an emissivity map. Here, this map was derived by a supervised object-oriented classification of a very high-resolution multispectral satellite image. The presented results demonstrate the sensitivity of the model to each one of the mentioned elements. In particular, the most critical aspect is the identification 
of the appropriate values of the surface emissivity; in fact, a decrease by $1 \%$ only of these values produces an average increase of the retrieved temperatures by about $0.3{ }^{\circ} \mathrm{C}$. Furthermore, the accurate mapping of the roofing materials and the proper registration of this map with the thermal image require considerable investments.

The sensitivity of the model to all of the mentioned factors and the fact that not all of the required ancillary information is always available prove how important the execution of ground surveys is to obtain reliable results. For the experimentations presented here, a full validation of the results with a statistically-significant number of ground control points (where the surface temperature is directly measured at the time of the flight) is still not possible. However, the comparison with five ground control points suggests that a precision of $1{ }^{\circ} \mathrm{C}$ is still difficult to achieve in complex environments.

Applying the data produced by the calibrated thermal imagery, a model to estimate the energy performance of building quarters can be developed and integrated into a spatial decision support system. For this purpose, additional information is needed (e.g., geometric data or the applied energy source); these data are unfortunately not all available in the majority of the real cases. For a quick decision support system without an extremely high need of additional data, a compromise is inevitable with respect to accuracy. In any case, models at the district level are generally more appropriate than at the individual building level.

In addition to that, the airborne thermal imagery can be an efficient tool for detecting hot spots and thermal bridges or for comparing roofs.

It is also to be noted that such tools do not substitute energy experts, but can facilitate their work and make their decisions faster.

\section{Acknowledgments}

The publication is realized in the framework of the TÁMOP-4.2.2.A-11/1/KONV-2012-0041 project. The project is co-financed by the European Union and the European Social Fund.

The data used for the experimentations presented here were acquired during the EnergyCity Project, founded by the Central Europe Program. The authors are grateful to Franco Coren and Rita Blanos of the National Institute of Oceanography and Experimental Geophysics (OGS) for providing the thermal images, to Siro Martello of the Corvallis Company for the SDSS development and to Massimo Bider and Sandro Nanni of ARPA (Regional Agency for Environmental Protection in the Emilia-Romagna Region) for providing the radiosonde data. The authors would like to thank also the Municipality of Bologna, in particular Andrea Minghetti, Paola Africani and Elisa Paselli of the "SIT" division, for providing the cartography and the LiDAR data, and the "Environment and Energy" division, for their support during the surveys. Finally, the authors would like to thank Alessia Orio for her contribution to the mosaicking of the thermal images.

\section{Author Contributions}

Gabriele Bitelli: coordination and overall article editing. Paolo Conte: ground surveys and image processing. Tamas Csoknyai: energetic modelling. Francesca Franci: supervised the classification of roofing materials. Valentina A. Girelli: thermal mosaic orthorectification. Emanuele Mandanici: atmospheric correction and software development. 


\section{Conflicts of Interest}

The authors declare no conflicts of interest.

\section{References}

1. Ürge-Vorsatz, D.; Novikova, A. Potentials and costs of carbon dioxide mitigation in the world's buildings, Energy Policy 2008, 36, 642-661.

2. Hay, G.J.; Kyle, C.; Hemachandran, B.; Chen, G.; Rahman, M.M.; Fung, T.S.; Arvai, J.L. Geospatial technologies to improve urban energy efficiency. Remote Sens. 2011, 3, 1380-1405.

3. Weng, Q.; Quattrochi, D.A. Thermal Remote Sensing of Urban Areas, Remote Sens. Environ. 2006, 104, 119-256.

4. Bitelli, G.; Conte, P. Techniques, tools and workflow for remote sensed roof temperature mapping in some cities across Central Europe. In Proceedings of the 17th International Conference "Building Services, Mechanical and Building Industry Days"-Urban Energy Conference, Debrecen, Hugary, 13-14 October 2011; pp. 49-56.

5. Voogt, J.A.; Oke, T.R. Thermal remote sensing of urban climates, Remote Sens. Environ. 2003, 86, 370-384.

6. Weng, Q. Thermal infrared remote sensing for urban climate and environmental studies: Methods, applications, and trends. ISPRS J. Photogramm. Remote Sens. 2009, 64, 335-344.

7. Hay, G.J.; Hemachandran, B.; Chen, G.; Kyle, C. HEAT-Home energy assessment technologies: A web2.0 residential waste heat analysis using geobia and airborne thermal imaginary. In Proceedings of the ISPRS Conference GEOBIA 2010: Geographic Object-Based Image Analysis, Ghent, Belgium, 29 June-2 July, 2010; Volume XXXVIII-4/C7.

8. Stockton, G.R.; Tache, A. Advances in applications for aerial infrared thermography. Proc. SPIE 2006, 6205, doi:10.1117/12.669513.

9. Allinson, D. Evaluation of aerial thermography to discriminate loft insulation in residential housing. Ph.D. Thesis. University of Nottingham, Nottingham, UK, 2007.

10. EnergyCity Project Website. Available online: http://www.energycity2013.eu/ (accessed on 22 January 2015).

11. Egeresi, M.; Hess, N.; Csoknyai, T. Integration of the building typology and the decision support system in the EnergyCity project. In Proceedings of the 16th Building Services, Mechanical and Building Industry Days International Conference: Urban Energy Conference, Debrecen, Hungary, 14 October 2011; pp. 57-64.

12. Byrnes, A.E.; Schott, J.R. Correction of thermal imagery for atmospheric effects using aircraft measurement and atmospheric modeling techniques. Appl. Opt. 1986, 25, 2563-2570.

13. Liang, S. Quantitative Remote Sensing of Land Surfaces; John Wiley \& Sons: Hoboken, NJ, USA, 2004.

14. Tang, B.H.; Wu, H.; Li, C.; Li, Z.L. Estimation of broadband surface emissivity from narrowband emissivities. Opt. Express 2011, 19, 185-192.

15. Popovic, M.J.; Kavran, J. Energy efficiency and renewal of residential buildings stock. Int. J. Contemp. Architect. "The New ARCH” 2014, 1, 93-100. 
16. Joint EPISCOPE and TABULA Website. Available online: http://episcope.eu/building-typology/ (accessed on 21 January 2015).

17. Berk, A.; Anderson, G.P.; Acharya, P.K.; Bernstein, L.S.; Muratov, L.; Lee, J.; Fox, M.J.; Adler-Golden, S.M.; Chetwynd, J.H.; Hoke, M.L.; et al. MODTRAN5: A reformulated atmospheric band model with auxiliary species and practical multiple scattering options. Proc. SPIE 2005, 5655, doi:10.1117/12.578758.

18. Jiménez-Muñoz, J.C.; Sobrino, J.A.; Mattar, C.; Franch, B. Atmospheric correction of optical imagery from MODIS and reanalysis atmospheric products. Remote Sens. Environ. 2010, 114, 2195-2210.

19. Sobrino, J.A.; Oltra-Carrió, R.; Jiménez-Mũnoz, J.C.; Julien, Y.; Sòria, G.; Franch, B.; Mattar, C. Emissivity mapping over urban areas using a classification-based approach: Application to the Dual-use European Security IR Experiment (DESIREX). Int. J. Appl. Earth Observ. Geoinf. 2012, $18,141-147$.

20. Pisello, A.L.; Cotana, F.; Nicolini, A.; Brinchi, L. Development of clay tile coatings for steep-sloped cool roofs. Energies 2013, 6, 3637-3653.

21. Hang, Y.; Lifu, Z.; Yingqian, G.; Shunshi, H.; Xueke, L.; Genzhong, Z.; Qingxi, T. Temperature and emissivity separation from Thermal Airborne Hyperspectral Imager (TASI) data. Photogramm. Eng. Remote Sens. 2013, 79, 1099-1107.

22. Lillesand, T.M.; Kiefer, R.W.; Chipman, J.W. Remote Sensing and Image Interpretation, 5th ed.; John Wiley \& Sons: Hoboken, NJ, USA, 2004.

23. Spronken-Smith, R.A.; Oke, T.R. The thermal regime of urban parks in two cities with different summer climates. Int. J. Remote Sens. 1998, 19, 2085-2104.

24. Offerle, B.; Eliasson, I.; Grimmond, C.S.B.; Holmer, B. Surface heating in relation to air temperature, wind and turbulence in an urban street canyon. Bound.-Lay. Meteorol. 2007, 122, 273-292.

25. Artis, D.A.; Carnahan, W.H. Survey of emissivity variability in thermography of urban areas. Remote Sens. Environ. 1982, 12, 313-329.

26. Dash, P.; Göttsche, F.-M.; Olesenm, F.-S.; Fischer, H. Land surface temperature and emissivity estimation from passive sensor data: Theory and practice-current trends. Int. J. Remote Sens. 2002, 23, 2563-2594.

27. Sobrino, J.A.; Jiménez-Muñoz, J.C.; Zarco-Tejada, P.J.; Sepulcre-Cantó, G.; de Miguel, E. Land surface temperature derived from airborne hyperspectral scanner thermal infrared data. Remote Sens. Environ. 2006, 102, 99-115.

28. Jacob, F.; Gu, X.F.; Hanocq, J.F.; Tallet, N.; Baret, F. Atmospheric corrections of single broadband channel and multidirectional airborne thermal infrared data: Application to the ReSeDA experiment. Int. J. Remote Sens. 2003, 24, 3269-3290.

(C) 2015 by the authors; licensee MDPI, Basel, Switzerland. This article is an open access article distributed under the terms and conditions of the Creative Commons Attribution license (http://creativecommons.org/licenses/by/4.0/). 